\title{
The obtaining of the Label Reliability Measure for gravity measurement by the LSBB underground lab
}

\author{
Camille Gonnet ${ }^{1}$, Claire Barra ${ }^{2}$, Jean-Baptiste Decitre ${ }^{2}$, Séverine Rosat ${ }^{3}$, Daniel Boyer ${ }^{2}$ \\ ${ }^{1}$ Centre de Technologie Méditerranéen de Métrologie (CT2M), route de Lançon - Centre des Creusets, 13250 Saint-Chamas, France \\ ${ }^{2}$ Laboratoire Souterrain à Bas Bruit (LSBB URL), La gra , France \\ ${ }^{3}$ Ecole et Observatoire des Sciences de la Terre (EOST), 5 rue René Descartes, 67084 Strasbourg, France
}

\begin{abstract}
The Low-Noise Underground Research Laboratory (LSBB URL) in Rustrel (near Avignon, Southern France) is born from the reco

1996, the site has been transformed in an international scientific facility for interdisciplinary research. Scientists working on different fields and from different countries are supervised by the engineer team of the LSBB. The LSBB URL wanted to point the competences of the lab and prove that their measures could be trusted. The accreditation according to the ISO 17025 didn't appear suitable. Indeed, this well-known standard is not really suited to the needs of research lab and the work to set up for a first accreditation is consistent and costly. Working with partners in the field of public and private research, the CT2M has developed a new quality system fully compatible with the ISO 17025 but more suitable to establish with the concerned academic labs and private companies: The "Label Fiabilité Mesure". The LSBB URL has obtained the graduation for the Label for its activity of measurement of gravity.
\end{abstract}

\section{The LSSB URL and the MIGA project}

\subsection{Presentation of the LSBB}

The Low Background Noise Underground Research Laboratory of Rustrel in the Vaucluse (nearby Avignon, Southern France, http://www.lsbb.eu) is a lab with worldwide unique specificities. Indeed, it is born in 1996 from the reconversion of the command firing post of the French deterrent ground-launched nuclear missiles. It has been built to be able to receive the launching presidential order within the room called "capsule".

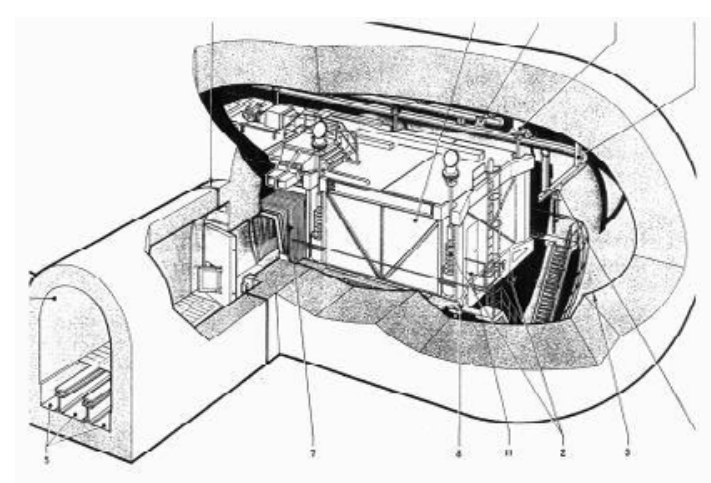

Fig. 1. The "Capsule" within a shielded room

The room has been built $500 \mathrm{~m}$ underground. It is hung in the air thanks to 8 shock-absorbers and protected by an enclosure compose of concrete and steel. It was designed to protect the person in charge for executing the launch of nuclear weapons.

Since 1996, it has been transformed in an international scientific facility for interdisciplinary research. The lab is composed of $3,7 \mathrm{~km}$ of gallery dug in cretaceous limestones and going down until $500 \mathrm{~m}$ below the mountain. On the one hand, LSBB URL gives in situ allowing research in geological and hydrogeological specific environment, on the other hand, its untypical design warrants electromagnetic as much as anthropological low background noise environment well adapted for testing and developing new high sensitivity instrumentation.

\subsection{MIGA project}

In partnership with the EOST (Strasbourg, France), the LSBB URL takes part in the European scientific project MIGA (Matter wave - laser based Interferometer Gravitation Antenna). The purpose of this project is the construction of a novel infrastructure to study strain tensor of space-time and gravitation. Using a novel approach for

MIGA project targets the construction of sensors useful for the environment monitoring and should help improving the mitigation of natural hazards. Located , the LSBB environment allows the measurement of gravitation gradient induced by the

\footnotetext{
* Corresponding author: cgonnet@ct2m.fr
}

(C) The Authors, published by EDP Sciences. This is an open access article distributed under the terms of the Creative Commons Attribution License 4.0 (http://creativecommons.org/licenses/by/4.0/). 
Newtonian noise. Therefore, it has been decided to install a high sensitivity cryogenic gravimeter there.

\section{The measurement of gravity}

\subsection{Gravimetry}

The superconducting gravimeter installed in the deeper LSBB vault allows continuous recording of the gravity. Gravity is affected by several factors. It depends on its location because of the multi-scale inhomogeneity of the Earth and of the atmosphere. It is also affected by dynamic and transitory phenomena like tides, seismic activities and human activity which produce local gravity variations.

Setting a relative gravimeter in the LSBB allows to record the variation of gravity due to natural dynamic phenomena since the gravimeter is protected from any other human perturbation. The measurement of " $g$ " contribute to study the earth structure and geological processes.

\subsection{Relative gravimeter}

The gravimeter setting in LSBB URL is a relative superconducting gravimeter made up with a sphere of Niobium of $28 \mathrm{~g}$ held in a magnetic levitation thanks to a permanent current running in two coils. This magnetic force balances the weight of the sphere and allows it to levitate. That is realized at low temperature around $4 \mathrm{~K}$ to make it very stable. The variation of the position of the sphere enables the measurement of " $g$ " with an accuracy closed to the $0.1 \mathrm{~nm} / \mathrm{s}^{2}$.

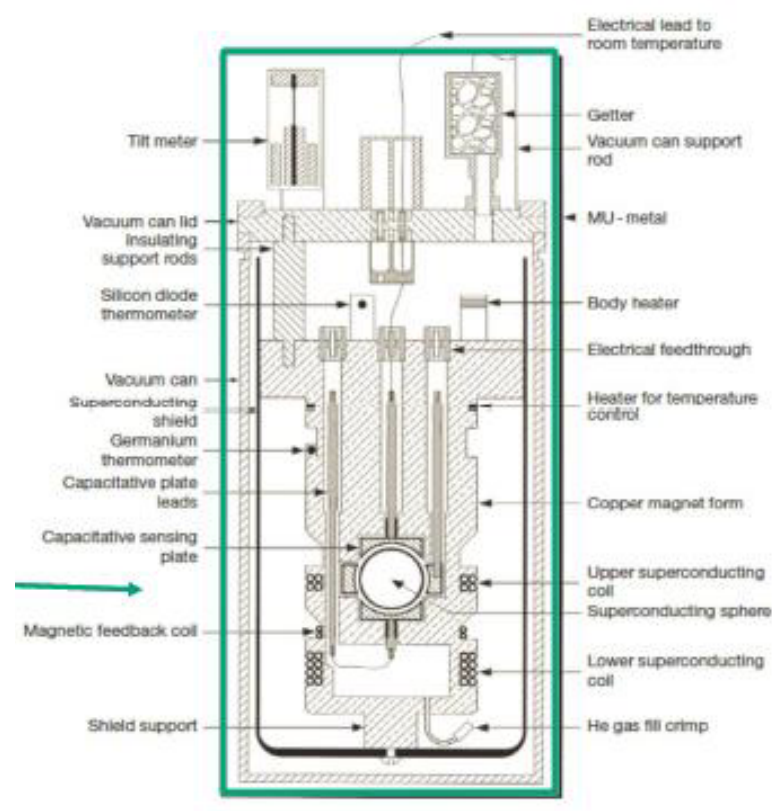

Fig. 2. Scheme of a relative superconducting gravimeter

The LSBB is responsible of the management of the superconducting gravimeter and the recording of the data. The LSBB controls the access to the room to reduce human impact on the measures. The data are then published on a public website.

\subsection{Absolute gravimeter}

To ensure metrological traceability, the relative gravimeter is calibrated by comparison with an absolute gravimeter. EOST realizes the calibration once a year and follows the drift of the equipment.

Absolute gravimeter measures the gravity from the free fall of a cube in vacuum. It is accomplished thanks to a Michelson interferometer. The first mirror is a face of a cube in free fall, the second is fixed and represents the reference. When the cube falls the optical path $(\mathrm{x})$ reduces with time (t) measured with an atomic clock. Thus, solving the equation of movement (Eq. 1), " $g$ " can be determined with an uncertainty associated to $x_{0}$ and $v_{0}$.

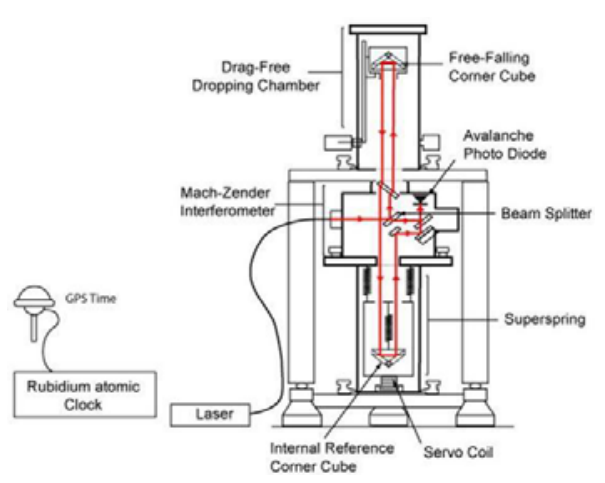

Fig. 3. Schematic drawing of an absolute gravimeter

Deviation of the superconducting gravimeter in Rustrel appeared to be very stable because of its specific location. The lab may be able to increase the calibration periodicity quickly.

\section{Setting of a quality system to showcase skills of the lab}

\subsection{The "Label Fiabilité Mesure", an alternative to the ISO 17025 for research labs}

The LSBB wanted to improve its quality level of measurements and get its technical skills recognized. First, accreditation according to ISO 17025 by the COFRAC was considered. But quickly it has appeared that the gap between the quality level of the LSBB and the quality level required by ISO 17025 was consistent.

Therefore, the lab has decided to go for another standard: the "Label Fiabilité Mesure" (Measurement Reliability Label). This alternative, proposed by the CT2M since 2011 (http://ct2m.fr), has been developed after discussions with partners in the field of public and private research (eg. INRA, CIRAD, CEA, CNRS, INRETS, STMicroelectronics, CEMAGREF, ...). It started from the fact that some requirements of ISO 17025 were not adapted for research laboratories and that the cost of accreditation was important. 


\subsection{Principle of the "Label Fiabilité Mesure"}

This standard, inspired by the ISO 17025 gather quality and technical requirements that stand on:

- The management of measure equipment,

- The control of measurement method (validation of methods, uncertainty estimation's),

- Metrological traceability,

- Staff training process,

- Participation to inter-laboratory comparison.

Two levels of label are possible. The first level is mostly adapted to research labs wishing to set up a quality system. It includes basic requirements considered as essential to guaranty reliability of measures.

The second level adds requirements in order to go further on the management of measurement system and on the setting up of a quality system. It rather aims to labs wishing to obtain a reconnaissance in their skills in terms of quality system and technical control of the measurement process.

The "Label Fiabilité Mesure" may also be seen as a steppingstone forward to COFRAC accreditation. The label is delivered after a first audit of the lab to ensure that all the requirements are followed. The conclusion of the inspection is then report to a committee that decides to award the lab or not. The label is maintained for 3 years sending a self-evaluation form to the CT2M before being fully re-inspected.

\subsection{Conclusion: The labelling of the LSBB URL}

The LSBB decided to get the "Label Fiabilité Mesure" for its gravity measures.

The process started in November 2015. A consultant of the CT2M came to visit the lab and made a first diagnosis. The LSBB applied directly to level II of the label. After, this meeting an action plan was transmitted to the lab to help and advise it.

One year later, on November 2016, the initial audit of label has been conducted by another consultant of the CT2M. The quality level of the LSBB had highly be improved. The LSBB obtained easily the level II of the label. Only one deviation was noticed and the LSBB gave quickly proves of its correction.

Daniel BOYER, deputy director of the LSBB testifies about the benefits cause by the label:

"The establishment of a standard as the label, in LSSB URL, is part of a multi-level approach. It allows to fix the technical and scientific framework for the gravimetric measure carried out by the LSBB. Thus, the sustainability is validated, the hazards are recorded and the measurement conditions are improved.

This approach aims to be extended to all the observation activities carried out on the site (seismology, tiltmetry, ...). The organization of the lab has also benefited from the labelling process thanks to the redaction of quality documents as procedure and to the harmonization of the documentation system."

\section{Acknowledgment}

Authors thank CNRS/DR12 for funding the work-linked training between LSBB URL and CESI School in Aix-enProvence in coordination with CT2M. Authors thank also Véronique Lacoste from IRSN for their fruitful recommendations and comments during labelling process. 